Journal of Statistical Planning and Inference, 2004, Vol. 126, No. 2, pp.553-568.

ISSN (print 0378-3758) (online 1873-1171)

DOI: $10.1016 /$ j.jspi.2003.08.008

http://www.elsevier.com/wps/find/journaldescription.cws home/505561/description\#description

http://www.sciencedirect.com/science?_ob=MImg\&_imagekey=B6V0M-49TJJT6-6-

4F\&_cdi=5650\&_user $=3366836 \& \_$orig=browse $\& \_c o v e r D a t e=12 \% 2 F 01 \% 2 F 2004 \& \_s k=998739997 \& v i e w=c \& w c h$

$\mathrm{p}=\mathrm{dGLbVlW-zSkzk \& md5=95f1 \textrm {c } 1 5 4 0 \mathrm { db } 2 8 \mathrm { b } 6 5 \mathrm { d } 3 7 8 8 \mathrm { ff } 0 \mathrm { c } 3 8 \mathrm { a } 3 1 \mathrm { b } 6 \& \mathrm { ie } = / \text { sdarticle.pdf }}$

(C) Elsevier Science, All rights reserved.

\title{
Statistical modeling and computer simulation of intergranular corrosion growth in AA2024-T3 aluminum alloy
}

\author{
Shiling Ruan ${ }^{a}$, Douglas A. Wolfe ${ }^{a}$, Gerald S. Frankel ${ }^{b}$ \\ ${ }^{a}$ Department of Statistics, Ohio State University, Columbus, $\mathrm{OH}$ \\ ${ }^{\mathrm{b}}$ Department of Materials Science and Engineering, Ohio State University, Columbus, $\mathrm{OH}$
}

\begin{abstract}
An extension of a brick wall model developed in a previous paper (Technometrics, to appear) was used to describe intergranular corrosion in AA2024-T3 aluminum alloys. The extended model simulates the behavior of corrosion paths at intersections of grain boundaries within the metal sample. Situations considered include the cases where a corrosion path might assume an upward turn, skip an intersection (not turn) or split into branches. The splitting of a corrosion path results in a smaller median of the minimum order statistic while the other factors increase the median of the minimum order statistic. Moreover, a larger number of grain layers increases the minimum path length for a sample with given thickness. With a proper combination of these factors, the extended model is able to provide a good fit to the experimental data developed by the foil penetration technique.
\end{abstract}

\section{Introduction}

High strength aluminum alloys such as AA2024-T3 are widely used in aerospace applications. They are resistant to uniform corrosion but highly susceptible to localized corrosion. Localized corrosion, usually in the forms of intergranular corrosion (IGC), pitting corrosion, crevice corrosion, exfoliation and stress corrosion cracking (Davis, 1999), is unpredictable in terms of the exact places of initiation and initiation time. With traditional deterministic approaches, such as the electrochemical theory of corrosion, localized corrosion cannot be well explained due to the scattering of the corrosion data. On the other hand, considering localized corrosion as rare events, statistical approaches could provide an appropriate way to describe the mechanism of corrosion (Shibata, 1996), potentially to evaluate quantitatively localized corrosion behavior.

Among all the forms of localized corrosion in high strength aluminum alloys in aqueous environment, IGC and pitting attack are two common forms that have received a good deal of attention. IGC is a preferential attack of grain boundaries or nearby adjacent regions without appreciable attack of the grain matrix, while pitting corrosion occurs at the intermetallic particles or in the grain matrix. Both forms of attack are similar from an electrochemical point of view (Galvele and De Micheli, 1970; Muller and Galvele, 1977). However, IGC might have very different growth kinetics from pitting. For predictive modeling of corrosion propagation, it is important to understand these growth kinetics independently. In this paper, we describe a model predicting the growth kinetics of IGC in aluminum alloy.

There are many factors that determine the resistance and susceptibility of an alloy to IGC, 
such as alloy composition, microstructure, and the environment (Davis, 1999; Scully et al., 1992; Scully, 1999). The exact role of each of these factors is still unclear. For example, even though there are a few reports on quantitative measurements of IGC in aluminum alloys, little is known about the relationship between alloy microstructure and IGC growth kinetics. Zhang and Frankel (2000) made quantitative measurements of localized corrosion kinetics in AA2024-T3 using the foil penetration technique. They reported that the growth kinetics of localized corrosion in this type of alloy exhibit a strong anisotropy as a result of anisotropy in the microstructure of the wrought aluminum alloy. AA2024-T3 has a typical laminated structure with grains elongated in the longitudinal (rolling) and long transverse directions relative to their dimension in the short transverse (through-thickness) direction. The time for intergranular corrosion to penetrate a given distance along the longitudinal or long transverse direction is much less than the time to penetrate the same nominal distance in the short transverse direction (Zhang and Frankel, 2000). The ratio of nominal penetration rates for the longitudinal direction to that for the short transverse direction was found by Zhang to be 4.29. The local intergranular growth rate should not depend on the direction of growth, though it is likely a function of total path length from the surface exposed to the bulk solution. The difference in nominal growth rate with throughthickness direction relative to the rolling direction is a result of the anisotropic grain dimensions and the resulting difference in path length. Any intergranular path in the through thickness direction of a plate with an elongated microstructure will be very convoluted, resulting in nominal rate of penetration that is much less than the local rate of intergranular growth. It is of interest to be able to determine the influence of a grain structure with a particular size and shape anisotropy on the kinetics of intergranular growth in the through thickness direction.

Ruan et al. (2004) proposed a statistical model to describe the relationship between the microstructure and the IGC growth rate based on foil penetration data and quantification of the microstructure of AA2024-T3. In the model, a brick wall represents the laminated microstructure of AA2024-T3. The distributions of the grain size (both width and length) are approximated by gamma distributions. Since the grain size in the longitudinal or rolling direction is much larger than that in the long transverse direction, the problem can be simplified into two dimensions, the short and long transverse dimensions. IGC in the longitudinal direction is assumed not to contribute to penetration in the short transverse direction. Given the length and the width of the grain, the distance that a corrosion path travels along a given grain is assumed to be uniformly distributed. Then, a Matlab program was used to simulate the distribution of the minimum order statistic of the corrosion path length. The simulation gives estimates with a small amount of underestimation compared to the actual result from Zhang's (2001) experiments.

The brick wall model relates the growth kinetics of AA2024-T3 aluminum alloy to the microstructure of the alloy. It provides a simple way to quantitatively evaluate the growth kinetics of IGC for a given microstructure in AA2024-T3. However, the brick wall model was based on a series of simplified assumptions, which do not provide a totally accurate description of the corrosion propagation process. In particular, there are two cases that were not accounted for in the model. First, corrosion was assumed to turn toward the bottom surface (away from the environment) at every intersection with a vertical grain boundary. However, a corrosion path might actually skip an intersection and not turn. Moreover, when a corrosion path does make a turn, it might turn up toward the top surface (toward the environment) or down toward the bottom surface of the metal strip depending on the nature of the three-way intersection. When a corrosion path turns upward and reaches the top surface, the propagation can be assumed to terminate. Second, a corrosion path was assumed not to split at any intersection while it might 
actually split into two corrosion paths at an intersection. Each of these two corrosion paths might propagate independently in the metal. Accordingly, the number of corrosion paths increases. Based on the above considerations of corrosion behavior, a more realistic brick wall model is discussed in this paper.

\section{Modeling corrosion growth at an intersection}

\subsection{Basic assumptions}

Consider a strip of metal with thickness $T$ and a total of $k$ grain layers across the thickness. The widths $b_{j}$ of the grains are taken to be common within a given layer but they are permitted to vary across different layers. That is,

$$
T=\sum_{j=1}^{k} b_{j} .
$$

Let $a$ denote grain length and assume that it has a distribution with pdf $f(a)$. As stated in the previous paper (Ruan et al., 2004), both the grain length and width are reasonably modeled by gamma distributions with appropriate parameter values $\alpha$ and $\beta$. Suppose there are $m$ corrosion

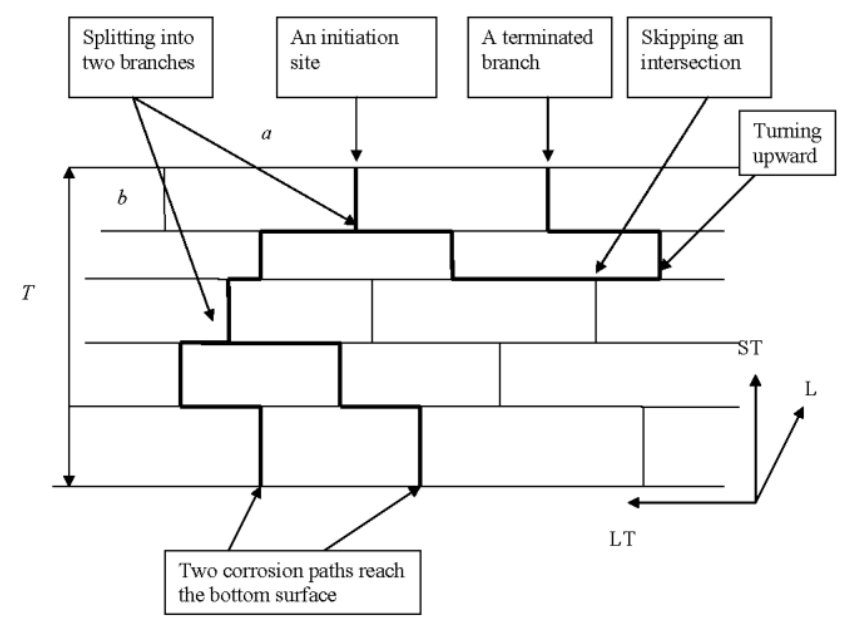

Fig. 1. A graphical representation of the growth behavior of a corrosion path at a three-way intersection in a strip of metal with $\mathrm{k}=5$ grain layers. The bold line represents $W_{i, D}$ and $a$ and $b$ are random variables representing the length and width, respectively, of a grain model. (Note: ST - short-transverse direction; LT-long-transverse direction; L-longitudinal direction.)

initialization points on the surface of the metal. For $i=1, \ldots, m$, let $W_{i, D}$ denote the distance that the $i$ th initial corrosion path travels to reach a fixed depth, say $D$, of the metal. If the corrosion path reaches the bottom surface, $W_{i, D}$ corresponds to $W_{i, T}$. Assume these $m$ corrosion paths are independent. Fig. 1 is a graphical representation of a brick wall model that represents an aluminum sample with a simplified layered microstructure. The corrosion path initiated from the top surface travels along a grain boundary that is perpendicular to the surface. It propagates 
along the grain boundary until it meets an intersection. Then, it might turn to a horizontal direction (either left or right on the figure), or it might split into two horizontal corrosion paths. In the former case, it propagates along the length direction of the grains until it meets another intersection. Depending on the nature of this new three-way intersection, it might turn upward, or downward, or might skip the intersection and continue propagation along the horizontal direction. Since the widths of the grains are small compared to the lengths of the grains, it is reasonable to assume that a corrosion path will always make a turn toward a horizontal direction at the end of a vertical step. If a corrosion path turns upward and reaches the top surface again, it is considered to be a terminated path since IGC corrosion does not propagate on the surface of the alloy. Additionally, it is assumed that a corrosion path cannot terminate anywhere except the top or bottom surface of the metal strip. In the case where a corrosion path splits into two horizontal pieces at an intersection, these pieces are viewed as two corrosion paths having initiated from the same place on the top surface with a common previous path length. These paths are then assumed to propagate independently in the remainder of the metal sample under the previously described assumptions. However, in the case of such a split, the total number of corrosion paths increases.

The foil penetration technique measures the time taken by the fastest corrosion growth path to reach the bottom surface. With the vital assumption that the local corrosion growth rate is identical in all directions, the fastest corrosion growth corresponds to the shortest corrosion path length (Zhang, 2001; Zhang et al, 2003). Correspondingly, any path that terminates before reaching the bottom surface should not be considered the shortest corrosion growth path (minimum order statistic) for our purposes.

When a horizontal corrosion path meets an intersection, it can either continue to propagate in the horizontal direction or turn toward a vertical direction that is perpendicular to the surface. There are two types of intersections, represented by " $T$ " and " $\perp$ ". For the " $\top$ " type intersection, the horizontal corrosion path can turn downward toward the bottom surface. For the "上" type intersection, the horizontal corrosion path can turn upward toward the top surface. Therefore, the probability that a horizontal corrosion path turns upward depends on the proportion of the " $\perp$ " type intersections among all the intersections it meets. Similarly, the probability that a corrosion path turns downward depends on the proportion of " $T$ " type intersections among all the intersections it meets. Let $p \perp$ and $p_{\top}$ denote these two proportions, respectively. Then,

$$
p_{\perp}+p_{\top}=1 .
$$

Let $p_{\text {skip }}$ represent the probability that a horizontal corrosion path skips an intersection and let $p_{\text {up }}$ and $p_{\text {down }}$ be the probabilities that it turns upward and downward, respectively. Then, according to our previous assumptions, we have

$$
p_{\text {up }}+p_{\text {down }}+p_{\text {skip }}=1 \text {. }
$$

If a horizontal corrosion path is known to make a turn at an intersection, the two conditional probabilities $p_{u \mathrm{p}} /\left(1-p_{\text {skip }}\right)$ and $p_{\text {down }} /\left(1-p_{\text {skip }}\right)$ describe the likelihood that a corrosion path would turn upward and downward, respectively, corresponding to the proportions of the " $\perp$ " and “T” types of intersections, respectively. That is, 


$$
\begin{aligned}
& p_{\perp}\left(1-p_{\text {skip }}\right)=p_{\text {up }}, \\
& p_{\top}\left(1-p_{\text {skip }}\right)=p_{\text {down }} .
\end{aligned}
$$

Next, let $p_{\text {split }}$ denote the probability that a corrosion path splits into two branches at an intersection at the end of a vertical step. We assume all of these probabilities are identical for every intersection.

We consider a total of $m$ initial corrosion sites on the top surface of a metal strip. Propagation with possible splitting results in $(m+u)$ path lengths, where $\mathrm{u} \geq 0$ is the total number of branches resulting from splitting of corrosion paths. Among these lengths, we let $v \geq$ 0 be the number of paths terminated on the top surface instead of the bottom surface. Therefore, the $(m+u-v)$ paths lead to a random number of corrosion path lengths and the minimum of these lengths is recorded as a random observation $W_{\min , T}$ from the distribution of the minimum path length for a metal strip of thickness $T$. The minimum order statistic for the corrosion path lengths is thus given by

$$
W_{\min , T}=\min _{i=1, \ldots, m+u-v} W_{i, T}, \quad i=1, \ldots, m+u-v,
$$

where $W_{i, T}$ is the length of the $i$ th corrosion path.

Let $W_{i, D \text {,horizontal }}$ and $W_{i, \mathrm{D} \text {,vertical }}$ represent the total horizontal distance and the total vertical distance, respectively, traveled by the $i$ th corrosion path, so that

$$
W_{i, D}=W_{i, D, \text { vertical }}+W_{i, D, \text { horizontal }}, \quad i=1, \ldots, m+u
$$

Let $T_{i}(j)$ be the vertical distance that the ith corrosion path travels along the width of the $j$ th grain before it turns toward a horizontal direction. That is,

$$
W_{i, D, \text { vertical }}=\sum_{j} T_{i}(j), \quad i=1, \ldots, m+u,
$$

where $j \in\{1,2, \ldots, k\}$, and $k$ is the total number of grain layers across the thickness. Since corrosion paths might turn upward and travel on previous layers again, the total vertical distance for a given corrosion path might not exactly equal the thickness $T$. Each $T_{i}(j)$ is, however, equal to the width of the $j$ th layer. Since the width of the grain in each layer is modeled by a gamma distribution, all the $T_{i}(j) s$ have a common gamma distribution. Let $D_{i}(j)$ represent the horizontal distance that the ith corrosion path travels on the bottom surface of the $j$ th layer of the metal, for $j$ $\in\{1, \ldots, k-1\}$, where $k$ is the total number of grain layers across the thickness. Note that no corrosion paths propagate on either the top surface of the first layer or on the bottom surface of the $k$ th layer. Then,

$$
W_{i, D, \text { horizontal }}=\sum_{j} D_{i}(j), \quad i=1, \ldots, m+u .
$$

When a corrosion path skips an intersection and keeps propagating in the horizontal direction, 
the associated $D_{i}(j)$ would include at least two horizontal pieces. In Fig. 2 we show such a situation where a corrosion path skips three successive intersection on the bottom surface of the first layer leading to four horizontal pieces that add up to $D_{i}(1)$. The corrosion path turns downward at the fourth intersection. (Note that a grain might be intercepted by other grains randomly on either the top or bottom surface.) Given the length of the grain, the first piece of $D_{i}(1)$ is modeled by uniform distribution and is denoted by $H_{i}(1)$. The unconditional distribution of this random variable was discussed in detail in Ruan et al. (2004). Briefly, the pdf, $h(d)$ of $H_{i}(1)$ is given by the following:

$$
\begin{aligned}
h(d) & =\int_{d}^{\infty} \frac{1}{a} \frac{1}{\Gamma(\alpha) \beta^{\alpha}} a^{\alpha-1} \mathrm{e}^{-a / \beta} \mathrm{d} a \\
& =\frac{1}{(\alpha-1) \beta} \int_{d}^{\infty} \frac{1}{\Gamma(\alpha-1) \beta^{\alpha-1}} a^{\alpha-2} \mathrm{e}^{-a / \beta} \mathrm{d} a, \quad 0<d<\infty,
\end{aligned}
$$

where a $\alpha>0$ and $\beta>0$ are the parameters of the gamma distribution used to model the distribution of grain lengths in the metal.

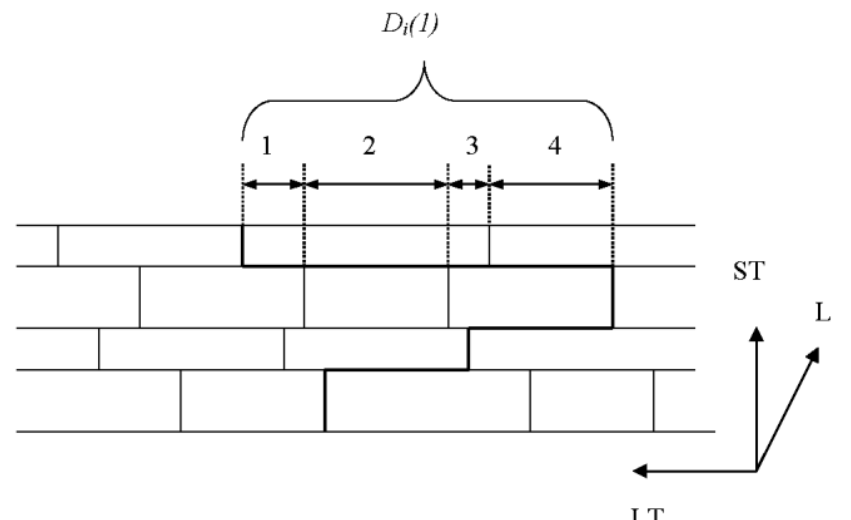

LT

Fig. 2. The bold line represents a corrosion path $W_{i, D} . D_{i}(1)$ is the horizontal distance that the ith corrosion path travels in the second grain layer.

Once a corrosion path skips an intersection, however, the remaining horizontal pieces on the grain layer are modeled solely by the gamma distribution without use of a conditional uniform distribution. We denote these pieces by $\left[G_{i}(1)\right]_{\mathrm{s}}, s=1, \ldots, n$, where $n$ is the total number of such complete horizontal pieces. We note that this is actually an upper bound approximation since the last piece of the horizontal distance might not cover an entire grain length before the path turns again. However, during propagation, we believe that a horizontal corrosion path is likely to meet many more " $T$ " type intersections than " $\perp$ " type intersections. Therefore, the probability that a corrosion path turns downward is likely to be greater than the probability that it turns upward. As a result, the upper bound approximation from using these complete horizontal gamma distances when a corrosion path skips an intersection should not result in serious overestimation. With this notation, we have

$$
D_{i}(1)=H_{i}(1)+\sum_{s=l}^{n}\left[G_{i}(1)\right]_{s}
$$




\subsection{Computer simulation}

Under the discussed assumptions, we used a Matlab algorithm to simulate the distribution of the minimum path length. First, the thickness of each layer, $b_{j}, j=1, \ldots, k$, is generated from a gamma distribution. The parameters of the gamma distribution are estimated via the method of moments (Ruan et al,, 2004). The sum of this set of random numbers is subject to the constraint $T=\sum_{\mathrm{j}=1}^{\mathrm{k}} b_{j}$. We must adjust the width of the last grain layer to accommodate.

For a corrosion path, the first step is always taken to be a vertical step $T_{i}(1)$, which is equal to $b_{1}$. Next, the first $H_{i}(1)$ from the distribution with pdf (2.9) is generated. Then, probabilities $p_{\text {up }}, p_{\text {down }}, p_{\text {skip }}$ and $p_{\text {split }}$ are assigned. As an example, consider $p_{\text {down }}=0: 8$ and $p_{\text {skip }}$ $=0: 1$ so that $p_{\text {up }}=1-p_{\text {down }}-p_{\text {skip }}=0: 1$ by (2.3). A random number $w$ is generated from the uniform $(0,1)$ distribution. If $w<0.1$, the corrosion assumes an upward turn. Accordingly, a vertical step is generated with the distance $b_{1}$, the thickness of the first grain layer. If $w>0.9$, the corrosion skips the intersection and a random number $G_{i},(1)$ is generated. If $w$ falls between 0.1 and 0.9, the corrosion path assumes a downward turn and the vertical step takes the value of $b_{2}$, the thickness of the second grain layer. An indicator variable, $X$, is used to record the layer number that the corrosion path is currently on. The initial value of $X$ is zero. When the corrosion path makes a downward turn, $X$ is increased by 1 . When the corrosion path makes an upward turn, $X$ is reduced by 1 . Otherwise, $X$ retains its current value. $X=0$ (except initially) corresponds to a corrosion path that is terminated at the top surface. Similarly, $X=k$ if a corrosion path reaches the bottom surface. The minimum path length is obtained from those corrosion paths that reach the bottom surface.

Starting from the first vertical step, it is necessary to consider whether a corrosion path might split into two branches. For example, assume $P_{\text {split }}=0: 2$. A random number $r$ is generated from the uniform $(0,1)$ distribution. If $r<0: 2$, the corrosion path is split into two horizontal pieces. Each of the branches is then simulated separately from this point on. The total number of branches and the number of the current layer where the splitting occurs are recorded. For branch 1 , the horizontal and vertical distances it travels are simulated accordingly given the known probabilities $p_{\text {up }}, p_{\text {down }}, p_{\text {skip }}$ and $p_{\text {split. }}$ If there is another split somewhere along the path, the layer number and the number of total splits are again recorded. After branch 1 reaches the bottom surface or terminates at the top surface, the program starts to simulate branch 2 . This branch has a portion overlapping with the first one, so the new simulation starts from the layer where the split occurs until the second branch is also terminated. This entire procedure is repeated until all of the branches have been simulated.

The entire set of corrosion paths constitutes a random sample from the distribution of $W_{i, D}, i=1, \ldots, m+u$. Using the indicator variable $X$ which records the current layer of the corrosion path, a random sample is generated from the distribution of $W_{i, T}, i=1, \ldots, m+u-v$; that is, from the distribution of corrosion path lengths that reach the bottom surface. The number of corrosion initialization sites, $m$, is estimated to be in the order of $10^{3}$ for this type of aluminum foil penetration samples (Ruan et al., 2004; Zhang et al., 2003). However, $m$ is assumed to be 100 in this paper in order to reduce the amount of computation and still illustrate the application of the simulation procedure. The minimum of these lengths is recorded as a random observation $W_{\min , T}$ from the distribution of the minimum path length. A sufficient number (e.g., sample size $=100$ ) of minimum path length values are generated by repeating the above procedure. The algorithm of the computer program is summarized in Fig. 3. 


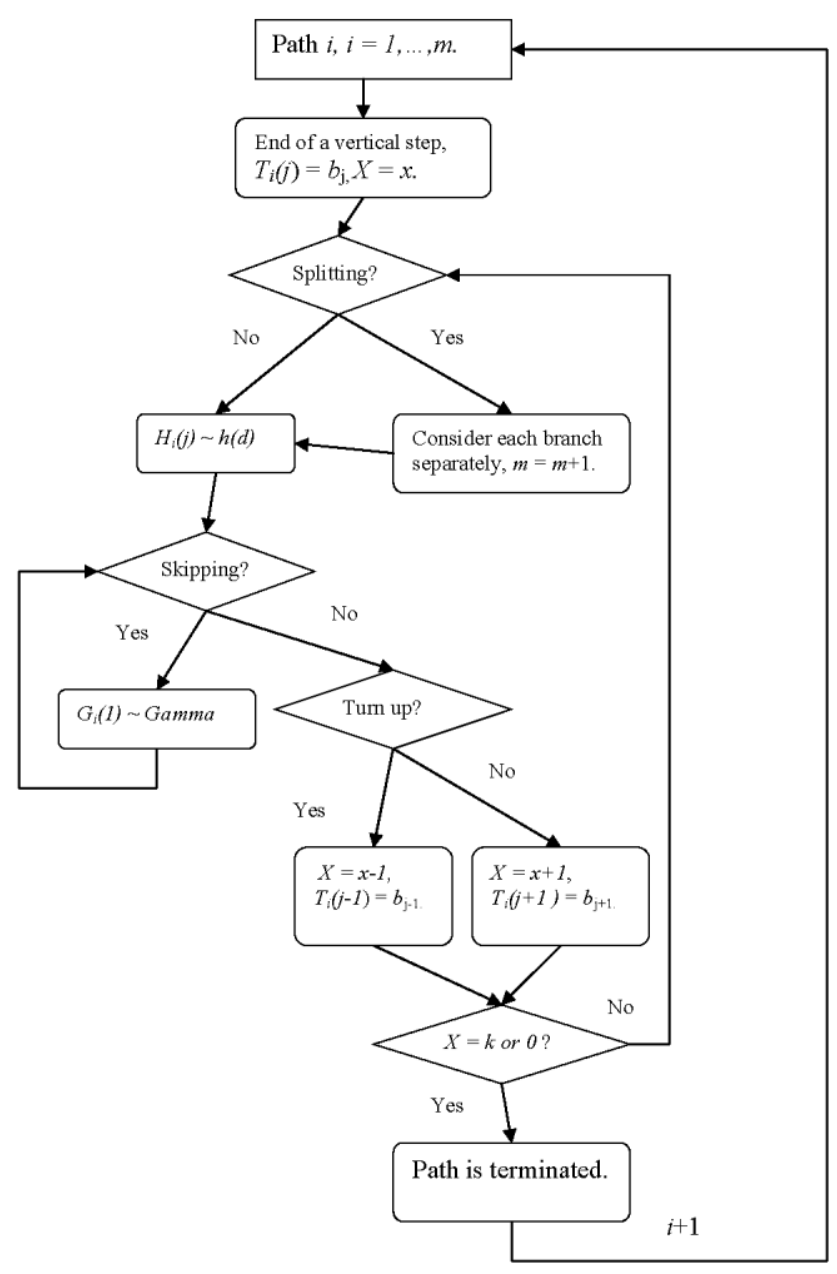

Fig. 3. A flow chart representing the computer simulation algorithm.

\section{Simulation results and discussion}

We use the method of moments to estimate the parameters of the gamma distributions in order to simulate the grain sizes. From previous work (Ruan et al., 2004), the method of moments estimators for the parameters $a$ and $\beta$ for grain length of the AA2024-T3 sample tested by Zhang (2001) are 4 and 0.075 , respectively. In addition, from Zhang (2003), the sample mean and standard deviation of the grain thickness measurements are 0.05 and 0:032 $\mathrm{mm}$, respectively. Assuming that the thickness of the grains is distributed as a gamma $\left(a^{\prime} \beta^{\prime}\right)$ distribution, it follows from the method of moments that solving

$$
a^{\prime} \beta^{\prime}=0.05
$$

and

$$
a^{\prime} \beta^{\prime 2}=0.032^{2}
$$


simultaneously yields $\hat{\alpha}^{\prime}=2: 44$ and $\hat{\boldsymbol{\beta}}^{\prime}=0: 02$. However, since $a^{\prime}$ must be an integer for the gamma distribution in our model, we could use either gamma $(2 ; 0: 0255)$ or gamma $(3 ; 0: 017)$ to simulate the distribution of grain thickness. In the case that a corrosion path can only assume a downward turn, these two sets of parameters give close results in terms of the median from the distribution of the minimum path length, as shown in Table 1 . In the case when $p_{\text {up }}=0: 05, p_{\text {skip }}=$ $0: 01$ and $p_{\text {split }}=0: 03$, however, the agreement between these two sets of parameters is not as good as the previous case, since the median for gamma $(2 ; 0: 0255)$ is 1.325 while the median for gamma $(3 ; 0: 017)$ is 1.385 . This small difference is most likely due to the randomness of the simulation rather than the difference in the parameters, i.e., the number of times that a corrosion path skips an intersection or splits into two pieces is different from path to path. Hence, either gamma $(2 ; 0: 0255)$ or gamma $(3 ; 0: 017)$ can be used to model the distribution of the grain width. For the rest of our study, we use only gamma (2;0:0255) to simulate the distribution of the grain thickness.

Using the algorithm described in Section 2.2, the influences of turning upward, skipping an intersection and splitting into two branches on the minimum corrosion path lengths are investigated separately and the results are summarized in Tables $2-4$, respectively. The thickness of the grain is simulated by a gamma $(2 ; 0: 0255)$ distribution. The number of corrosion

Table 1

Comparison of the results for gamma $(2 ; 0: 0255)$ and gamma $(3 ; 0: 017)$

\begin{tabular}{lll} 
& $\begin{array}{l}\text { Median of minimum path } \\
\text { length }\left(p_{\text {down }}=1, p_{\text {up }}=0,\right.\end{array}$ & $\begin{array}{l}\text { Median of minimum path length } \\
\left(p_{\text {down }}=0.94, p_{\text {up }}=0.05, p_{\text {skip }}=\right. \\
\left.0.01, p_{\text {split }}=0.03\right)(\mathrm{mm})\end{array}$ \\
\hline$\left.p_{\text {skip }}=0, p_{\text {split }}=0\right)(\mathrm{mm})$ & 1.325 \\
Gamma $(2,0.0255)$ & 1.296 & 1.385 \\
\hline Gamma $(3,0.017)$ & 1.294 & .
\end{tabular}

The number of sums is $m=100$ and the number of layers is $k=12$. A random sample of size 100 was taken from the distribution of the minimum path length for each model.

Table 2

Simulation results for minimum corrosion path length when a corrosion path can turn upward or downward but not skip an intersection or split into branches $\left(p_{\text {skip }}=0 ; p_{\text {split }}=0\right)$

\begin{tabular}{lllll} 
Sample & $p_{\text {down }}$ & $p_{\text {up }}$ & $\begin{array}{l}\text { Median of minimum path } \\
\text { length }(M)(\mathrm{mm})\end{array}$ & Normalized ratio \\
\hline 1 & & & 1.296 & 3.24 \\
2 & 1.00 & 0.00 & 1.358 & 3.39 \\
3 & 0.95 & 0.05 & 1.438 & 3.59 \\
4 & 0.90 & 0.10 & 1.485 & 3.71 \\
5 & 0.85 & 0.15 & 1.580 & 3.95 \\
6 & 0.80 & 0.20 & 1.701 & 4.25 \\
7 & 0.75 & 0.25 & 1.828 & 5.37 \\
8 & 0.70 & 0.30 & 2.123 & 5.69 \\
9 & 0.65 & 0.35 & 2.275 & 6.97 \\
10 & 0.60 & 0.40 & 2.787 & 9.22 \\
11 & 0.55 & 0.45 & 3.686 & \\
\hline
\end{tabular}


Table 3

Simulation results for minimum corrosion path length when a corrosion path can turn downward or skip an intersection but not turn upward or split into branches $\left(p_{\text {up }}=0 ; p_{\text {split }}=0\right)$

\begin{tabular}{lllll} 
Sample & $p_{\text {down }}$ & $p_{\text {skip }}$ & $\begin{array}{l}\text { Median of minimum path } \\
\text { length }(M)(\mathrm{mm})\end{array}$ & $\begin{array}{l}\text { Normalized } \\
\text { ratio }\end{array}$ \\
\hline 12 & 0.95 & 0.05 & 1.362 & 3.40 \\
13 & 0.90 & 0.10 & 1.435 & 3.59 \\
14 & 0.85 & 0.15 & 1.486 & 3.72 \\
15 & 0.80 & 0.20 & 1.586 & 3.96 \\
16 & 0.75 & 0.25 & 1.701 & 4.25 \\
17 & 0.70 & 0.30 & 1.812 & 4.53 \\
18 & 0.65 & 0.35 & 1.955 & 4.89 \\
19 & 0.60 & 0.40 & 2.124 & 5.31 \\
20 & 0.55 & 0.45 & 2.246 & 5.61 \\
21 & 0.50 & 0.50 & 2.488 & 6.22 \\
\hline
\end{tabular}

Table 4

Simulation results for minimum corrosion path length when a corrosion path can split into two branches at the end of a vertical step, but not turn upward or skip an intersection $\left(p_{\text {up }}=0 ; p_{\text {skip }}=0\right)$

\begin{tabular}{lllll} 
Sample & $p_{\text {down }}$ & $p_{\text {split }}$ & $\begin{array}{l}\text { Median of } \\
\text { minimum path } \\
\text { length }(M)(\mathrm{mm})\end{array}$ & Normalized ratio \\
\hline 22 & 0.95 & 0.05 & 1.232 & 3.08 \\
23 & 0.90 & 0.10 & 1.196 & 2.99 \\
24 & 0.85 & 0.15 & 1.154 & 2.89 \\
25 & 0.80 & 0.20 & 1.142 & 2.86 \\
26 & 0.75 & 0.25 & 1.092 & 2.73 \\
27 & 0.70 & 0.30 & 1.054 & 2.63 \\
28 & 0.65 & 0.35 & 1.042 & 2.60 \\
29 & 0.60 & 0.40 & 1.016 & 2.54 \\
30 & 0.55 & 0.45 & 0.991 & 2.48 \\
31 & 0.50 & 0.50 & 0.960 & 2.40 \\
\hline
\end{tabular}

initialization sites is $m=100$, the number of layers is $k=12$, and the sample thickness $T$ is assumed to be $0: 4 \mathrm{~mm}$. A random sample of size 100 is taken from the distribution of the minimum path length and the median of these observations $M$ is computed, along with the normalized ratio, given by the expression

$$
\text { Normalized ratio }=\frac{M}{T}
$$

This ratio is expected to be close to 4.29 for the sample of AA2024-T3 tested by Zhang (2001).

Table 2 summarizes the simulation results for the setting where a corrosion path can turn 
up or down but not skip or split at an intersection $\left(p_{\text {skip }}=0 ; p_{\text {split }}=0\right)$. The probability of turning

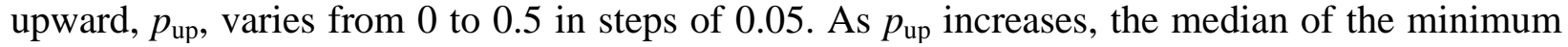
path length tends to increase. For $p_{\text {up }}$ between 0 and 0.5 , the median $M$ increase is roughly linear

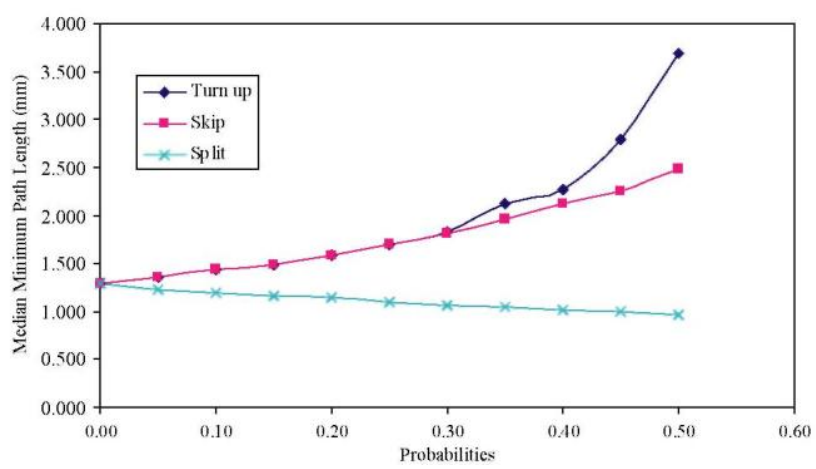

Fig. 4. Effect of probabilities $\left(p_{u p}, p_{\text {skip }}, p_{\text {split }}\right)$ on the median of the minimum path length.

in the range of 0.1-0.3 but the increase is more dramatic for $p_{\text {up }}>0: 3$ (Fig. 4). When $p_{\text {up }}=0$, some underestimation exists in the estimated median minimum path length because the normalized ratio is smaller than the target 4.29. When $p_{\text {up }}$ is large, the model overestimates this nominal median minimum path length as the normalized ratio increases dramatically. The increase in simulated median minimum path length can be attributed to two factors. When a corrosion path assumes an upward turn, it propagates along a more tortuous route than those paths that do not turn upward. On the other hand, some corrosion paths that turn upward might terminate at the top surface of the metal strip, thus decreasing the total number of paths reaching the bottom surface. In this case, the minimum order statistic is likely increased. However, the influence of $m$ on minimum order statistic is small (Zhang et al., 2003).

Table 3 summarizes the simulation results for the setting where a corrosion path can assume a downward turn at an intersection or skip the intersection but it cannot turn upward or split $\left(p_{\text {up }}=0 ; p_{\text {split }}=0\right)$. The probability of skipping an intersection, $p_{\text {skip }}$, varies from 0.05 to 0.5 in steps of 0.05 . As $p_{\text {skip }}$ increases, the median of the minimum path length tends to increase. We note that when the two probabilities $p_{\text {up }}$ and $p_{\text {skip }}$ are small, they have similar influence on both the median of the minimum path length and the normalized ratio. They demonstrate similar amounts of random variation with slightly increasing trends. When both probabilities are large, $p_{\text {up }}$ is more influential than $p_{\text {skip, }}$ as clearly shown in Fig. 4. When a corrosion path skips an intersection and continues to propagate in the horizontal direction, the total horizontal distance it travels will increase. However, when a corrosion path assumes an upward turn, both its horizontal distance and vertical distance traveled will increase. Additionally, $p_{\text {skip }}$ does not have the potential to decrease the number of corrosion paths that reach the bottom surface as does $p_{\text {up }}$.

Table 4 summarizes the simulation results for the setting where a corrosion path can split into two branches at an intersection at the end of any vertical step but it cannot turn upward or skip an intersection $\left(p_{\text {up }}=0 ; p_{\text {skip }}=0\right)$. As with other settings, the probability of splitting at an 


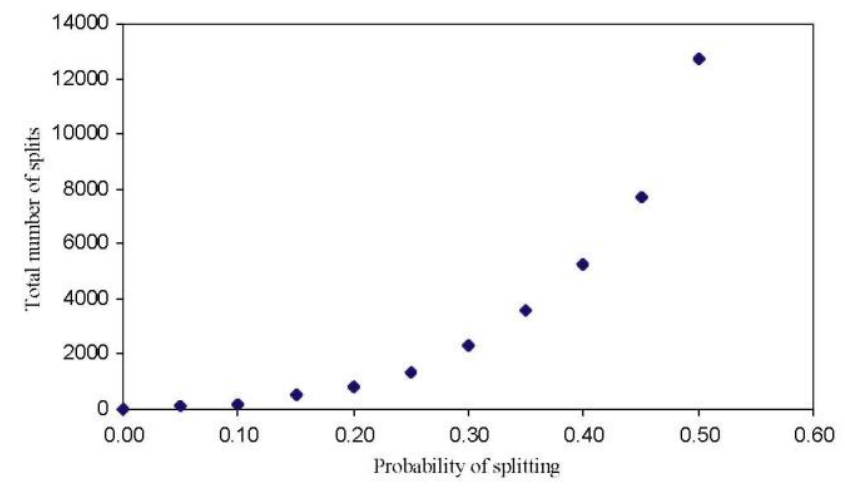

Fig. 5. Effect of the total number of splits on the median minimum path length.

intersection, $p_{\text {split, }}$, varies from 0.05 to 0.5 in steps of 0.05 . As $p_{\text {split }}$ increases, the median of the minimum path length tends to gradually decrease linearly (Fig. 4). The influence of $p_{\text {split }}$ is

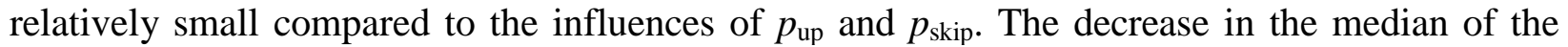
minimum path length is due to the fact that the minimum order statistic is likely to decrease as the number of paths reaching the bottom surface increases. In the case that $p_{\text {up }}$ and $p_{\text {skip }}$ are both equal to zero, the total number of paths that reach the bottom surface is the sum of the number of initial corrosion sites $m$ and the number of splits that occurred during corrosion propagation. However, the effect of the total number of paths on the minimum order statistic is relatively small compared to the effects of $p_{\text {up }}$ and $p_{\text {skip. }}$. Fig. 5 shows a representative sample of the number of splits that occurred for each $p_{\text {split. }}$ As $p_{\text {split }}$ increases from 0.05 to 0.5 , the observed number of splits changes from a magnitude of $10^{2}$ to $10^{4}$, but the variation in the median of the minimum path length is less than 0:3 mm, as shown in Table 4.

When the horizontal step is allowed to split into two pieces, one that keeps propagating horizontally and the other that turns to a vertical direction, the total number of paths increases. However, compared to the case where only vertical steps are allowed to split, the effect of the added number of splits by the splitting of the horizontal direction on the minimum order statistic does not provide additional interesting information. There are two explanations. First, when $p_{\text {split }}$ is small, the total number of splits is small and the number of splits added by the splitting of horizontal pieces is also very small. Therefore, the decrease in the minimum order statistic due to a slightly larger number of paths is small. When $p_{\text {split }}$ is larger, the number of splits increases substantially for both vertical steps and horizontal steps, from the magnitude of $10^{2}$ to the order of $10^{4}-10^{5}$. With such larger number of paths, the decrease in the minimum order statistic levels off. Therefore, the number of splits by adding the splitting of the horizontal paths also does not significantly affect the minimum order statistic. These simulation results agree with previous comments by Zhang (2001). In conclusion, our previous assumption that a horizontal step is not allowed to split does provide sufficient information about the influence of the splitting of corrosion paths on the minimum order statistic. 
Table 5

Effect of increases in the number of layers on the median of the minimum order statistic $\left(p_{\text {down }}=0: 94 ; p_{\text {up }}=\right.$ $\left.0: 05 ; p_{\text {skip }}=0: 01 ; p_{\text {split }}=0: 05\right)$. Sample thickness $T=0: 4 \mathrm{~mm}$

\begin{tabular}{lll} 
Number of layers & $\begin{array}{l}\text { Median of minimum path } \\
\text { length, } M\end{array}$ & Normalized ratio \\
\hline 8 & 0.932 & 2.33 \\
9 & 1.004 & 2.51 \\
10 & 1.130 & 2.83 \\
11 & 1.251 & 3.13 \\
12 & 1.325 & 3.31 \\
\hline
\end{tabular}

Table 6

Simulation results for combinations of probabilities ( $\left.p_{\text {up }}, p_{\text {skip }}, p_{\text {split }}\right)$. Sample thickness $\mathrm{T}=0: 4 \mathrm{~mm}$

\begin{tabular}{lllllll}
\hline Sample & $p_{\text {down }}$ & $p_{\text {up }}$ & $p_{\text {skip }}$ & $p_{\text {split }}$ & $\begin{array}{l}\text { Median of } \\
\text { minimum path } \\
\text { length }(M)(\mathrm{mm})\end{array}$ & $\begin{array}{l}\text { Normalized } \\
\text { ratio }\end{array}$ \\
\hline 32 & 0.85 & 0.05 & 0.1 & 0.1 & 1.348 & 3.37 \\
33 & 0.8 & 0.1 & 0.1 & 0.1 & 1.395 & 3.49 \\
34 & 0.75 & 0.15 & 0.1 & 0.1 & 1.531 & 3.83 \\
35 & 0.7 & 0.2 & 0.1 & 0.05 & 1.799 & 4.50 \\
36 & 0.7 & 0.2 & 0.1 & 0.1 & 1.703 & 4.32 \\
37 & 0.7 & 0.2 & 0.1 & 0.15 & 1.728 & \\
\hline
\end{tabular}

In addition to the behavior of a corrosion path at intersections, the number of layers in a metal strip is another influential factor that affects the minimum path length. The more layers there are in a metal strip, the smaller will be the thickness of the grains. Clearly, the minimum path length is an increasing function of the number of grain layers since that means more intersections within the metal strips. Thus, there are more opportunities for corrosion paths to turn up or skip an intersection. Of course, this also provides more opportunities to split into two branches, but, as noted, the effect of splitting is much less than the effect of either turning up or skipping. For example, suppose metal strips of the same thickness (e.g., $T=0: 4 \mathrm{~mm}$ ) are used for the simulated experiment. All other parameters are the same as described previously except the strips have different numbers of grain layers. The simulation results are summarized in Table 5. It is interesting to note that the median $M$ is increased by approximately 0.1 when the number of layers increases by 1 . In this model, the number of layers is variable because the grain thickness exhibits a distribution. In reality, the variability in the number of layers for a sample of a give thickness is small.

For a given metal strip, the number of layers is fixed. Therefore, we can adjust the probabilities ( $p_{\text {up }}, p_{\text {skip }}, p_{\text {split }}$ ) to provide combinations that yield good fits to the data. This can be useful in providing information about the possible corrosion behaviors in the metal. The following are simulation results for several combinations of probabilities. Comparing the normalized ratio to 4.29, it seems that the samples 36 and 37 in Table 6 provide a good fit to the data. Such a combination is not unique. As mentioned, the $p_{\text {up }}$ and $p_{\text {down }}$ are related to the chances that a corrosion path meets " $\perp$ " and " $T$ " types of intersections and $p_{\text {up }}$ is much smaller than $p_{\text {down. }}$. It seems that for given $p_{\text {up }}$ and $p_{\text {down }}, p_{\text {skip }}$ and $p_{\text {split }}$ can be adjusted to get different normalized ratios. However, estimation of these probabilities is not yet available from experiments. 


\section{Conclusions}

In this paper, we discuss an extension of the brick wall model proposed by Ruan et al. (2004). The basic brick wall model underestimates the minimum path length that a corrosion path travels along grain boundaries in an aluminum alloy sample. This problem is addressed by modeling the behavior of corrosion paths at intersections of grain boundaries. Situations considered include the cases where a corrosion path might assume an upward turn, skip an intersection or split into branches. We found that small percentage changes in the probabilities of any of these options can result in significant changes in the median of the minimum order statistic and the normalized ratio. However, with a proper combination of these probabilities, the extended model is able to obtain a good fit to the experimental data. This extension of the brick wall model represents a more precise description of the growth kinetics for AA2024-T3.

Even though it is still unknown in practice what values are reasonable to assign to these probabilities for this type of alloy, the simulation of such phenomena can provide useful quantitative insights into the understanding of the corrosion kinetics in AA2024-T3. If deemed necessary for a given metal alloy, further refinement of this model is also possible. For example, a corrosion path might have positive probabilities to turn to one direction or split into two branches no matter whether it is at a horizontal or vertical step. That is, even at the end of a horizontal step the corrosion path might split into two branches, where one branch skips the intersection and the other turns to a vertical direction. Also, it is reasonable to allow a corrosion path to terminate within the metal when it meets another corrosion path from an opposite direction.

\section{Acknowledgements}

S. Ruan and D.A. Wolfe were supported by the National Science Foundation under Award Number DMS9802358. G.S. Frankel was supported by the United States Air Force through a grant from S\& K Technologies. We thank the referees and the associate editor for their comments, which led to an improved presentation of our results.

\section{References}

Davis, J.R. (Ed.), 1999. Corrosion of Aluminum and Aluminum Alloys. ASM International, Materials Park, OH.

Galvele, J.R., De Micheli, S.M., 1970. Mechanism of intergranular corrosion of Al-Cu alloys. Corrosion Science $10,795-807$.

Muller, I.L., Galvele, J.R., 1977. Pitting potential of high purity binary aluminum alloys-I, Al-Cu alloys. Pitting and intergranular corrosion. Corrosion Science 17, 179-193.

Ruan, S., Wolfe, D.A., Zhang, W., Frankel, G.S., 2004. Statistical modeling of minimum intergranular corrosion path length in high strength aluminum alloy. Technometrics, to appear. Scully, J.R., Peebles, D.E., Romig, A.D., Frear, D.R, Hills, C.R., 1992. Metallurgical factors influencing the corrosion of aluminum, Al-Cu, and Al-Si alloy thin-films in dilute hydrofluoric solution. Metallurgical Transactions A - Physical Metallurgy and Material Science 23, 2641-2655.

Scully, J.R., 1999. Environment-assisted intergranular cracking. Materials Research Society Bulletin 24,36-42. Shibata, T., 1996. Statistical and stochastic approaches to localized corrosion. Corrosion 52 (11), 813-830.

Zhang, W., 2001. Localized corrosion and its kinetics in high strength Al alloy. Ph.D. Dissertation, Ohio State University, Department of Materials Science and Engineering.

Zhang, W., Frankel, G.S., 2000. Anisotropy of localized corrosion in AA2024-T3. Electrochemical and Solid State Letters 3, 268-270.

Zhang, W., Ruan, S., Wolfe, D.A., Frankel, G.S., 2003. Statistical model for intergranular corrosion growth kinetics. Corrosion Science 45, 353-370. 